hep-th/9411109

TUTP-94-18

November 1994

\title{
One-Loop Renormalization of a Self-Interacting Scalar Field in Nonsimply Connected Spacetimes
}

\author{
L.H.Ford and N.F.Svaiter \\ Institute of Cosmology, Department of Physics and Astronomy \\ Tufts University, Medford, Massachusetts 02155 USA
}

\begin{abstract}
Using the effective potential, we study the one-loop renormalization of a massive self-interacting scalar field at finite temperature in flat manifolds with one or more compactified spatial dimensions. We prove that, owing to the compactification and finite temperature, the renormalized physical parameters of the theory (mass and coupling constant) acquire thermal and topological contributions. In the case of one compactified spatial dimension at finite temperature, we find that the corrections to the mass are positive, but those to the coupling constant are negative. We discuss the possibility of triviality, i.e. that the renormalized coupling constant goes to zero at some temperature or at some radius of the compactified spatial dimension.
\end{abstract}

*Permanent address: Centro Brasileiro de Pesquisas Fisica-CBPF, Rua Dr. Xavier Sigaud 150, Rio de Janeiro, RJ 22290-180, Brazil 


\section{Introduction}

It is well known that one loop quantum corrections may alter the physical parameters of an interacting quantum field theory. In general this alteration is not of a form which can be absorbed by a simple redefinition of the parameters, in the way that one can remove the ultraviolet divergences. A simple example of this occurs in finite temperature field theory, where the renormalized mass can become temperature dependent [1]. Similarly, in flat spacetime with compactification in one spatial direction, the mass can depend upon the periodicity length in the compact direction [2, 3, 汭. This phenomenon is of particular interest in theories with broken symmetry, as it allows both thermal and topological effects to play a role in the breaking and restoration of symmetry [5]. The aim of this paper is to discuss quantum field theory at finite temperature in a spacetime where at least one of the spatial dimensions is compactified. The particular model which we adopt is a scalar field with quartic self-coupling. In particular, we wish to investigate the dependence of the renormalized mass and coupling constant upon the temperature and the size of the compactified dimension. We will calculate the effective potential, which may be expressed in terms of Epstein zeta functions. The ultraviolet divergences may be removed by analytic regularization and renormalization.

The outline of this paper is the following. In Section II, periodic boundary conditions are imposed upon the fields (after a Wick rotation), and the temperature dependent one-loop effective potential is calculated. The theory is regularized using an analytic continuation of the inhomogeneous Epstein zeta function. The renormalization of $\lambda \varphi^{4}$ theory in this multiply connected spacetime can be done by introducing counterterms, and we show that the mass and coupling constant counterterms are temperature and size independent

In Section III, we assume finite temperature and only one compactified spatial dimension. We explicitly calculate the corrections to both the mass and the coupling constant in this case. We find that the corrections to the mass are positive but those to the coupling constant are negative. The results are discussed in Section IV. In particular, we discuss the possibility of arranging for the renormalized coupling constant to vanish ("triviality") at some particular temperature or spatial size. In this paper we use units in which $\hbar=c=k_{B}=1$. 


\section{The effective potential of a scalar field at finite temper- ature}

In this section we study a real massive scalar field at finite temperature, where we assume that the topology of the spacelike sections is that of a three-torus. This kind of topology allows two different types of scalar fields. One which is periodic in the identified spatial coordinates is called an untwisted field, and the other which is antiperiodic in the identified spatial coordinate is called a twisted scalar field[6]. To study twisted scalar fields, we cannot assume that the normalized vacuum expectation value of the field is constant and the effective potential cannot be used. For the sake of simplicity, in this paper we will study only the untwisted scalar field.

The Lagrange density of the field is

$$
\mathcal{L}=\frac{1}{2} \partial_{\mu} \varphi_{u} \partial^{\mu} \varphi_{u}-\frac{1}{2} m_{0}^{2} \varphi_{u}^{2}-\frac{\lambda_{0}}{4 !} \varphi_{u}^{4}
$$

where $\varphi_{u}(x)$ is the unrenormalized field, and $m_{0}$ and $\lambda_{0}$ are the bare mass and coupling constant, respectively. We may rewrite the Lagrange density in the usual form where the counterterms will appear explicitly. Defining

$$
\begin{gathered}
\varphi_{u}(x)=(1+\delta Z)^{\frac{1}{2}} \varphi(x) \\
m_{0}^{2}=\left(m^{2}+\delta m^{2}\right)(1+\delta Z)^{-1} \\
\lambda_{0}=(\lambda+\delta \lambda)(1+\delta Z)^{-2}
\end{gathered}
$$

and substituting Eqs. (2), (3) and (4) into Eq. (1), we have

$$
\mathcal{L}=\frac{1}{2} \partial_{\mu} \varphi \partial^{\mu} \varphi-\frac{1}{2} m^{2} \varphi^{2}-\frac{\lambda}{4 !} \varphi^{4}+\frac{1}{2} \delta Z \partial_{\mu} \varphi \partial^{\mu} \varphi-\frac{1}{2} \delta m^{2} \varphi^{2}-\frac{1}{4 !} \delta \lambda \varphi^{4}
$$

where $\delta Z, \delta m^{2}$, and $\delta \lambda$ are the wave function, mass and coupling constant counterterms of the model. Through this paper we will assume that $m^{2}>0$. In the one-loop approximation, the effective potential at zero temperature in uncompactified spacetime is given by [7, 8]

$$
V\left(\varphi_{0}\right)=\frac{1}{2} m^{2} \varphi_{0}^{2}+\frac{\lambda}{4 !} \varphi_{0}^{4}-\frac{1}{2} \delta m^{2} \varphi_{0}^{2}-\frac{1}{4 !} \delta \lambda \varphi_{0}^{4}
$$




$$
+\quad i \int d^{4} q \frac{1}{(2 \pi)^{4}} \sum_{s=1}^{\infty} \frac{1}{2 s}\left(\frac{1}{2} \lambda \varphi_{0}^{2}\right)^{s} \frac{1}{\left(q^{2}-m^{2}+i \epsilon\right)^{s}} .
$$

There is no difficulty in extending the above results to finite temperature states. In this case, functional integrals will run over the fields that satisfy periodic boundary conditions in Euclidian time. The effective action can be defined as in the zero temperature case by a functional Legendre transformation, and regularization and renormalization procedures follow the same steps as in the zero temperature case. Similarly, compactification in imposed by requiring that the field be periodic in the spatial directions.

It has been shown that for models where the spacelike section are noncompact, all the divergences present in the Feynman loops are temperature independent 9, 10]. Similarly, the renormalization of the zero temperature theory with at least one compactified spatial dimension has been investigated by Toms 11] and by Birrell and Ford 12. These authors found that through the two-loop level, all of the counterterms are independent of the spatial size. A more general discussion has been given by Banach 13], who shows that topological identifications will not introduce new counterterms. Thus the divergences of the theory are independent of both temperature and spatial size. If this were not the case, there would be a danger that the renormalizability of the theory would be upset by changing either the temperature or the spatial topology.

Let us assume that we have a massive scalar field at finite temperature $\beta^{-1}$, and that the spacelike section is compactified with the topology of a three torus of sides $L_{1}, L_{2}$ and $L_{3}$. Define

$$
\begin{gathered}
c^{2}=\frac{m^{2}}{4 \pi^{2} \mu^{2}} \\
(\beta \mu)^{2}=a_{4}^{-1} \\
\left(L_{i} \mu\right)^{2}=a_{i}^{-1} \quad i=1,2,3
\end{gathered}
$$

where $\mu$ is a mass parameter introduced to keep the Epstein zeta function a dimensionless quantity. The euclidean effective potential becomes

$$
\begin{aligned}
V_{E}\left(\beta, L_{1}, L_{2}, L_{3}, \varphi_{0}\right) & =\frac{1}{2} m^{2} \varphi_{0}^{2}+\frac{\lambda}{4 !} \varphi_{0}^{4}-\frac{1}{2} \delta m^{2} \varphi_{0}^{2}-\frac{1}{4 !} \delta \lambda \varphi_{0}^{4} \\
& +\frac{1}{\beta L_{1} L_{2} L_{3}} \sum_{s=1}^{\infty} \frac{(-1)^{s+1}}{2 s}\left(\frac{\lambda}{8 \pi^{2}}\right)^{s}\left(\frac{\varphi_{0}}{\mu}\right)^{2 s} A_{4}^{c^{2}}\left(s, a_{1}, a_{2}, a_{3}, a_{4}\right)
\end{aligned}
$$


where

$$
A_{N}^{c^{2}}\left(s, a_{1}, a_{2}, . ., a_{N}\right)=\sum_{n_{1}, n_{2} . . n_{N}=-\infty}^{\infty}\left(a_{1} n_{1}^{2}+a_{2} n_{2}^{2}+\ldots+a_{N} n_{N}^{2}+c^{2}\right)^{-s}
$$

is the inhomogeneous Epstein zeta function [14, 15]. Note that in going from Eq. (6) to Eq. (10), we have first performed a Wick rotation so that the momenta are euclidean, and then have replaced the momentum integrals by discrete sums. In the case $c^{2}=0$, Eq. (11) defines a Madelung sum in the theory of classical lattices. If we impose the condition that the renormalized mass is zero, there is a problem in defining the renormalized coupling constant. The way to circumvented this difficulty is to impose the renormalizations conditions not at $\varphi_{0}=0$ but at another point. For a careful discussion, see the paper by Coleman and Weinberg[7]. In this paper we assume $m^{2}>0$, and the above problem does not appear. In the limit $L_{i} \rightarrow \infty$, the expression given by Eq. (10) differs from the usual finite temperature effective potential by terms that are independent of $\varphi_{0}$ [16]. Because only derivatives with respect to $\varphi_{0}$ correspond to physically meaningful quantities, this does not pose any problems.

Let us define the modified inhomogeneous Epstein zeta function as

$$
E_{N}^{c^{2}}\left(s, a_{1}, a_{2}, . . a_{N}\right)=\sum_{n_{1}, n_{2}, . . . n_{N}=1}^{\infty}\left(a_{1} n_{1}^{2}+. .+a_{N} n_{N}^{2}+c^{2}\right)^{-s}
$$

A simple calculation gives

$$
\begin{aligned}
A_{4}^{c^{2}}\left(s, a_{1}, a_{2}, a_{3}, a_{4}\right) & =16 E_{4}^{c^{2}}\left(s, a_{1}, a_{2}, a_{3}, a_{4}\right)+8 E_{3}^{c^{2}}\left(s, a_{1}, a_{2}, a_{4}\right)+8 E_{3}^{c^{2}}\left(s, a_{1}, a_{3}, a_{4}\right) \\
& +8 E_{3}^{c^{2}}\left(s, a_{2}, a_{3}, a_{4}\right)+8 E_{3}^{c^{2}}\left(s, a_{1}, a_{2}, a_{3}\right)+4 E_{2}^{c^{2}}\left(s, a_{1}, a_{2}\right) \\
& +4 E_{2}^{c^{2}}\left(s, a_{1}, a_{3}\right)+4 E_{2}^{c^{2}}\left(s, a_{1}, a_{4}\right)+4 E_{2}^{c^{2}}\left(s, a_{2}, a_{3}\right) \\
& +4 E_{2}^{c^{2}}\left(s, a_{2}, a_{4}\right)+4 E_{2}^{c^{2}}\left(s, a_{3}, a_{4}\right)+2 E_{1}^{c^{2}}\left(s, a_{1}\right) \\
& +2 E_{1}^{c^{2}}\left(s, a_{2}\right)+2 E_{1}^{c^{2}}\left(s, a_{3}\right)+2 E_{1}^{c^{2}}\left(s, a_{4}\right)+c^{-2 s} .
\end{aligned}
$$

Defining the new coupling constant and a dimensionless vacuum expectation value of the field by

$$
\begin{aligned}
& g=\frac{\lambda}{8 \pi^{2}} \\
& \frac{\varphi_{0}}{\mu}=\phi,
\end{aligned}
$$


the finite temperature one-loop effective potential is given by

$$
\begin{aligned}
V_{E}\left(\beta, L_{1}, L_{2}, L_{3}, \phi\right) & =\mu^{4}\left(2 \pi^{2} c^{2} \phi^{2}+\frac{1}{3} \pi^{2} g \phi^{4}-\frac{1}{2 \mu^{2}} \delta m^{2} \phi^{2}-\frac{1}{4 !} \delta \lambda \phi^{4}\right) \\
& +\frac{1}{\beta L_{1} L_{2} L_{3}} \sum_{s=1}^{\infty} \frac{(-1)^{s+1}}{2 s} g^{s} \phi^{2 s} A_{4}^{c^{2}}\left(s, a_{1}, a_{2}, a_{3}, a_{4}\right) .
\end{aligned}
$$

It is possible to regularize the one-loop effective potential introducing a cutoff in the Euclidian region, but we prefer to use the method of analytic extension. Let us assume that each term of the series in $s$ in the one-loop effective potential $V_{E}\left(\beta, L_{1}, L_{2}, L_{3}, \varphi_{0}\right)$ is an analytic extension, defined in the beginning only in an open connected set. To render the discussion more general, let us discuss the process of the analytic continuation of the modified inhomogeneous Epstein zeta function given by Eq. (12). For $R e(s)>\frac{N}{2}$, the $E_{N}^{c^{2}}\left(s, a_{1}, a_{2}, . . a_{N}\right)$ converges and represent an analytic function of $s$, so $s>\frac{N}{2}$ is the largest possible domain of the convergence of the series. This means that in Eq. (10) only the terms $s=1$ and $s=2$ are divergent. The $s=1$ term arises from the self-energy diagram (the one-loop process with two external lines), and the $s=2$ term arises from the one-loop correction to the scattering amplitude (the one-loop diagram with four external lines). After regularization, we may think of the first two terms in the sum in Eq. (10) as being evaluated not at $s=1$ and $s=2$, but rather at $s=1+\alpha$ and $s=2+\alpha$, respectively, where $\alpha$ is a complex parameter which vanishes in the limit in which the regularization is removed.

Using a Melin transform, it is possible to continue analytically $E_{N}^{c^{2}}\left(s, a_{1}, . ., a_{N}\right)$ from $\operatorname{Re}(s)>\frac{N}{2}$ to $R e(s) \leq \frac{N}{2}$, although isolated singularities will appear in the closed region $R e(s) \leq \frac{N}{2}$ at the points

$$
s=\frac{N}{2}, \frac{N-1}{2}, \cdots \frac{1}{2},-\frac{2 l+1}{2}, \quad l \in N .
$$

At these points, the analytic extension of $E_{N}^{c^{2}}\left(s, a_{1} . ., a_{N}\right)$ has first order poles, with residues $\operatorname{Res}\left[E_{N}^{c^{2}}\left(s, a_{1}, . ., a_{N}\right), s_{i}\right]$. The exact expression of the residue at the points in which we are interested is

$$
\operatorname{Res}\left[E_{N}^{c 2}\left(s, a_{1}, \ldots, a_{N}\right), \frac{j}{2}\right]=\frac{(-1)^{N-j} \pi^{\frac{j}{2}}}{2^{N} \Gamma\left(\frac{j}{2}\right)} \sum_{k=0}^{\frac{N-j}{2}} \frac{(-1)^{k}}{k !} c^{2 k} \pi^{k} A(2 k+j)
$$

where

$$
A(k)=\sum_{\left\{i_{1}, . ., i_{k}\right\}} \sqrt{a_{i_{1}} \ldots a_{i_{k}}}
$$


and $\sum_{i_{1}, . . i_{k}}$ denotes the sum over all possible choices of the $i_{1}, . . i_{k}$ among $1, \ldots N$ (for $k=0$ the sum is set equal to one) [17]. An appropriate choice of $\delta m^{2}$ and $\delta \lambda$ will remove the poles at $s=1$ and $s=2$, respectively. The idea to continue analytically expressions and subtract the poles was exploited by various authors 18, 19. In the method used by Bollini, Giambiagi and Domingues 18, a complex parameter was introduced as an exponent of the denominator of the loop expressions and the integrals are well defined analytic functions of the parameter for some $s_{0}$. Performing an analytic extension of this expression for $s<s_{0}$, poles will appear in the analytic extension and the final expression becomes finite after the subtraction of the poles. It is clear that our regularization is exactly a discrete version of the Bollini et al analytic regularization.

In our problem, the renormalization conditions are given by

$$
\left.\frac{\partial^{2}}{\partial \phi^{2}} V_{E}\left(\beta, L_{1}, L_{2}, L_{3}, \phi\right)\right|_{\phi=0}=4 \pi^{2} \mu^{4} c^{2}=\mu^{2} m^{2}
$$

and

$$
\left.\frac{\partial^{4}}{\partial \phi^{4}} V_{E}\left(\beta, L_{1}, L_{2}, L_{3}, \phi\right)\right|_{\phi=0}=8 \pi^{2} \mu^{4} g=\mu^{4} \lambda
$$

Substituting Eq. (16) into Eqs. (20) and (21) in such a way that the counterterm $\delta m^{2}$ cancels the pole contribution at $s=1$ of the analytic extension of the inhomogeneous Epstein zeta function, and $\delta \lambda$ cancels the pole contribution at $s=2$, we have

$$
\delta m^{2}=\frac{g}{\beta L_{1} L_{2} L_{3} \mu^{2}} \frac{1}{s-1} \operatorname{Res}\left[A_{4}^{c^{2}}\left(s, a_{1}, a_{2}, a_{3}, a_{4}\right), s=1\right]
$$

and

$$
\delta \lambda=-24 \frac{g^{2}}{\beta L_{1} L_{2} L_{3} \mu^{4}} \frac{1}{s-2} \operatorname{Res}\left[A_{4}^{c^{2}}\left(s, a_{1}, a_{2}, a_{3}, a_{4}\right), s=2\right] .
$$

By substitution of Eq. (18) and Eq. (19) into Eq. (22) and Eq. (23), it is straightforward to show that both $\delta \lambda$ and $\delta m^{2}$ are temperature and size independent. This shows that in the oneloop approximation, the counterterms of the model are independent of the parameters which are associated with the nontrivial topology. Hence if the model is renormalizable at zero temperature with certain counterterms, it is also renormalizable at finite temperature with exactly the same counterterms. 


\section{Topological and Thermal Corrections to the Mass and Coupling Constant}

In this section we will investigate the thermal and topological correction to the renormalized mass and coupling constant in the case where there is compactification in only one spatial direction. Set $L=L_{3}$ and take the limit in which $L_{1} \rightarrow \infty$ and $L_{2} \rightarrow \infty$. The finite temperature one-loop effective potential in this case is given by:

$$
V_{E}(\beta, L, \phi)=\mu^{4}\left(2 \pi^{2} c^{2} \phi^{2}+\frac{1}{3} \pi^{2} g \phi^{4}-\frac{1}{2 \mu^{2}} \delta m^{2} \phi^{2}-\frac{1}{4 !} \delta \lambda \phi^{4}\right)+G_{E}(\beta, L, \phi)
$$

where

$$
G_{E}(\beta, L, \phi)=\mu^{4} \sqrt{a_{3} a_{4}} \sum_{s=1}^{\infty} \frac{(-1)^{s+1}}{2 s} g^{s} \phi^{2 s} \int d^{2} k A_{2}^{M^{2}}\left(s, a_{3}, a_{4}\right),
$$

In the above equation

$$
M^{2}=\left(k^{1}\right)^{2}+\left(k^{2}\right)^{2}+c^{2}
$$

where

$$
k^{1}=\frac{q^{1}}{2 \pi \mu}
$$

and

$$
k^{2}=\frac{q^{2}}{2 \pi \mu}
$$

are dimensionless quantities.

Using the identity

$$
\int \frac{d^{d} l}{\left(l^{2}+a^{2}\right)^{b}}=\frac{\pi^{d / 2}}{\Gamma(b)} \Gamma\left(b-\frac{d}{2}\right) a^{d-2 b},
$$

we can perform the integrations over the continuous momenta and write

$$
G_{E}\left(a_{3}, a_{4}, \phi\right)=\mu^{4} \sqrt{a_{3} a_{4}} \pi \sum_{s=0}^{\infty} \frac{(-1)^{s+2}}{2 s+2} g^{s+1} \phi^{2 s+2} \frac{\Gamma(s)}{\Gamma(s+1)} A_{2}^{c^{2}}\left(s, a_{3}, a_{4}\right) .
$$

Note that the poles which were at $s=1$ and $s=2$ in Eq. (10) are now located at $s=0$ and $s=1$, respectively, in Eq. (27). As before, we regularize Eq. (27) by analytically continuing the summand around the points $s=0$ and $s=1$. 
The formal correction to the squared mass is given by

$$
\Delta^{\prime} m^{2}=\left(\frac{d^{2} G_{E}}{d \varphi_{0}^{2}}\right)_{s=0}=\pi g \mu^{2} \sqrt{a_{3} a_{4}} \lim _{s \rightarrow 0}\left[\Gamma(s) A_{2}^{c^{2}}\left(s, a_{3}, a_{4}\right)\right] .
$$

The $s \rightarrow 0$ limit of the Epstein zeta function is evaluated in the Appendix. The result, Eq. (A6), may be used to separate $\Delta^{\prime} m^{2}$ into its infinite and finite parts:

$$
\Delta^{\prime} m^{2}=\delta m^{2}+\Delta m^{2}
$$

where $\delta m^{2}$ is the counterterm to be absorbed by mass renormalization, and $\Delta m^{2}$ is the finite correction to the mass. The latter is defined so as to vanish in the limit of zero temperature in noncompactified space. Explicitly, we have

$$
\delta m^{2}=g \mu^{2}\left(-\frac{\pi c^{2}}{s}+2 \pi \ln c\right)
$$

and

$$
\Delta m^{2}=\frac{\lambda}{4 \pi^{2}}\left[m^{2} \int_{1}^{\infty} \frac{\left(t^{2}-1\right)^{\frac{1}{2}} d t}{\mathrm{e}^{m \beta t}-1}-\frac{\pi}{\beta L} \sum_{n=-\infty}^{\infty} \ln \left(1-e^{-2 \pi L \beta^{-1} \sqrt{n^{2}+m^{2} \beta^{2} / 4 \pi^{2}}}\right)\right] .
$$

There is an equivalent expression obtained by interchange of $\beta$ and $L$. Note that $\Delta m^{2} \geq 0$ for all choices of the parameters. The first term in Eq. (31) is the purely thermal correction to the mass[1]. In the limit that $L \rightarrow \infty$, the second term vanishes, and this correction is all that survives. The second term becomes the purely topological correction[3] in the limit of zero temperature:

$$
\Delta m^{2} \sim-\frac{\lambda}{2 \pi L} \int_{0}^{\infty} d x \ln \left(1-e^{-2 \pi L \sqrt{x^{2}+m^{2} / 4 \pi^{2}}}\right)=\frac{\lambda}{4 \pi^{2}} m^{2} \int_{1}^{\infty} \frac{\left(t^{2}-1\right)^{\frac{1}{2}} d t}{\mathrm{e}^{m L t}-1}
$$

(An integration by parts was performed to obtain the last form.)

The formal correction to the coupling constant $\lambda$ due to finite temperature and/or spatial compactification is given by

$$
\Delta^{\prime} \lambda=\left(\frac{d^{4} G_{E}}{d \varphi_{0}^{4}}\right)_{s=1}=-\frac{3 \lambda^{2}}{32 \pi^{3}} \sqrt{a_{3} a_{4}} \lim _{s \rightarrow 1} A_{2}^{c^{2}}\left(s, a_{3}, a_{4}\right) .
$$

This quantity is, of course, ill-defined because of a pole term at $s=1$ which needs to be isolated and removed. In the Appendix, it is shown that

$$
A_{2}^{c^{2}}\left(s, a_{3}, a_{4}\right) \sim \frac{\pi}{\sqrt{a_{3} a_{4}}}\left[\frac{1}{s-1}-\ln c^{2}+\cdots\right]+F_{1}\left(a_{3}, a_{4}\right), \quad s \rightarrow 1 .
$$


The pole term is absorbed by the $\delta \lambda$ counterterm when we let

$$
\delta \lambda=-\frac{3 \lambda^{2}}{32 \pi^{2}}\left(\frac{1}{s-1}-\ln c^{2}\right)
$$

Then the finite correction to the coupling constant is given by

$$
\Delta \lambda=-\frac{3 \lambda^{2}}{32 \pi^{3}} \sqrt{a_{3} a_{4}} F_{1}\left(a_{3}, a_{4}\right)
$$

Here

$$
F_{1}\left(a_{3}, a_{4}\right)=\frac{1}{\sqrt{a_{3} a_{4}}}\left[f\left(a_{3}\right)+f\left(a_{4}\right)+R\left(a_{3}, a_{4}\right)\right]
$$

where

$$
f\left(a_{3}\right)=4 \pi \int_{1}^{\infty} \frac{\left(t^{2}-1\right)^{-\frac{1}{2}} d t}{\mathrm{e}^{2 \pi c t / \sqrt{a_{3}}}-1}
$$

and $R\left(a_{3}, a_{4}\right)$ is given by

$$
\begin{aligned}
R\left(a_{3}, a_{4}\right) & =2 \pi \sqrt{a_{3}} \sum_{n=-\infty}^{\infty} \frac{1}{\sqrt{a_{3} n^{2}+c^{2}}\left(e^{2 \pi \sqrt{\left(a_{3} n^{2}+c^{2}\right) / a_{4}}}-1\right)}-f\left(a_{4}\right) \\
& =2 \pi \sqrt{a_{4}} \sum_{n=-\infty}^{\infty} \frac{1}{\sqrt{a_{4} n^{2}+c^{2}}\left(e^{2 \pi \sqrt{\left(a_{4} n^{2}+c^{2}\right) / a_{3}}}-1\right)}-f\left(a_{3}\right) .
\end{aligned}
$$

Note that the thermal and topological corrections to the coupling constant are always negative:

$$
\Delta \lambda<0
$$

which follows, for example, from Eqs. (37-39), where it is apparent that $F_{1}\left(a_{3}, a_{4}\right)>0$. The three terms on the right-hand side of Eq. (36) can each be given a physical interpretation. The $f\left(a_{3}\right)$ term is the purely topological term. It is the correction to the coupling constant at zero temperature in a space with one compact dimension. Similarly, the $f\left(a_{4}\right)$ term is the purely thermal term, which is the correction to the coupling constant at finite temperature in uncompactified space. The $R$ term represents a coupling between thermal and topological effects which is present only at finite temperature in compactified space. 
It is of interest to examine the small mass limit of this correction to the coupling constant. In the limit that $m \rightarrow 0$, the dominant contribution to $\Delta \lambda$ comes from the $n=0$ term in $R\left(a_{3}, a_{4}\right)$, and we obtain

$$
\Delta \lambda \sim-\frac{3 \lambda^{2}}{8 m^{2} \beta L}, \quad m \rightarrow 0 .
$$

This result seems to indicate that the one loop correction to the coupling constant can be arbitrarily negative for small masses. However, one must be careful about the limits of validity of the one loop approximation. This issue will be discussed in more detail in the next section. Note that the coupling constant correction described by Eq. (41) is nontrivial only at finite temperature in compactified spacetime, i.e. when both $L$ and $\beta$ are finite.

Let us now consider the purely thermal correction in more detail. Let $L \rightarrow \infty$, so that $a_{3} \rightarrow 0$. Then

$$
\Delta \lambda=-\frac{3 \lambda^{2}}{32 \pi^{3}} f\left(a_{4}\right)=-\frac{3 \lambda^{2}}{8 \pi^{2}} \int_{1}^{\infty} \frac{\left(t^{2}-1\right)^{-\frac{1}{2}} d t}{\mathrm{e}^{\beta m t}-1} .
$$

In the low temperature $\operatorname{limit}(\beta \rightarrow \infty)$, we have

$$
\Delta \lambda \approx-\frac{3 \lambda^{2}}{8 \pi^{2}} \int_{1}^{\infty}\left(t^{2}-1\right)^{-\frac{1}{2}} \mathrm{e}^{-\beta m t} d t=-\frac{3 \lambda^{2}}{8 \pi^{2}} K_{0}(\beta m) \approx-\frac{3 \lambda^{2}}{8 \pi^{2}} \sqrt{\frac{\pi}{2 \beta m}} \mathrm{e}^{-\beta m} .
$$

Similarly, in the high temperature limit $(\beta \rightarrow 0)$, we may use

$$
\frac{1}{\mathrm{e}^{2 \pi \beta m t}-1} \approx \frac{1}{\beta m t}=\frac{T}{m t}
$$

to write

$$
\Delta \lambda \sim-\frac{3 \lambda^{2} T}{16 \pi m}, \quad T \rightarrow \infty .
$$

Again, this correction would seem to be large in the case that $T \gg m$.

In this section we have found that in a space with one compact spatial dimension and/or at finite temperature, the one-loop correction to the squared mass is always positive, whereas that to the coupling constant is always negative. In the limits that either the temperature vanishes, or that the size of the compact dimension becomes large, we recover the results of previous authors for the mass correction, $\Delta m^{2}$. To our knowledge, the results presented here for the coupling constant correction, $\Delta \lambda$, have not been given before. The only reference of which we are aware which 
discusses either thermal or topological corrections to coupling constants is Higuchi and Parker 20]. Of course, all of the results of this section also apply to the case of a spacetime with periodicity in two spatial directions, but at zero temperature. One simply replaces $L$ and $\beta$ in the above formulas by $L_{1}$ and $L_{2}$, the two periodicity lengths.

\section{Discussion}

In this paper, we have calculated the mass correction, $\Delta m^{2}$, and the coupling constant correction, $\Delta \lambda$, due to both finite temperature and compactification in one spatial direction. We found that $\Delta m^{2} \geq 0$, whereas $\Delta \lambda \leq 0$. One of the primary reasons for interest in $\Delta m^{2}$ is its role in symmetry restoration. It had been noted by previous authors that $\Delta m^{2} \geq 0$ when one has either finite temperature in uncompactified space, or compactification at zero temperature. Thus in both cases, the effect of the radiative correction is to restore broken symmetries. Our results show that this effect also holds when one has a finite temperature state in compactified space. The extension of these results to models in spacetime dimensions other than four and to other model field theories is undertaken in a separate paper 21.

An interesting feature of the negative coupling constant correction is that it tends to make the theory less strongly coupled. One is then tempted to raise the question of whether it would even be possible to cause the net coupling constant to vanish, i.e., to achieve triviality at some particular temperature or compactification length. We have defined $\lambda$ to denote the renormalized coupling constant at zero temperature in uncompactified space. Thus the effective coupling constant when either $L$ or $\beta$ are finite is

$$
\lambda^{\prime}=\lambda+\Delta \lambda
$$

The one-loop correction, $\Delta \lambda$, is of order $\lambda^{2}$, so it is not clear that one can make it equal to $\lambda$ in magnitude before the one-loop approximation fails. The crucial issue here is just what are the limits of validity of this approximation. If it is simply that one needs $\lambda \ll 1$, then this does not prevent us from arranging a situation where $\lambda^{\prime}=0$. This is apparent from Eqs. (41) or (45). However, it is not clear that the true expansion parameter is $\lambda$ itself. It could be $\lambda$ multiplied 
by a dimensionless function of $m, L$, and $T$. If, for example, the limit of validity of Eq. (45) is when $\lambda T / m \ll 1$, then it is not possible to use this relation at the point where $\lambda^{\prime}$ would vanish. To settle this question, it would be necessary to have a reliable estimate of the magnitude of the higher order corrections.

\section{Acknowledgement}

We would like to thank Prof. A. Vilenkin for several helpful discussions. N.F. Svaiter would like to acknowledge the hospitality of the Institute of Cosmology, Tufts University, where part of this work was carried out . This work was supported by Conselho Nacional de Desenvolvimento Cientifico e Tecnologico do Brazil (CNPq) and by National Science Foundation Grant PHY-9208805.

\section{Appendix}

In this appendix, we wish to derive an expression for the Epstein zeta function $A_{2}^{c^{2}}\left(s, a_{1}, a_{2}\right)$. This quantity is initially defined by the double sum

$$
A_{2}^{c^{2}}\left(s, a_{1}, a_{2}\right)=\sum_{n_{1}, n_{2}=-\infty}^{\infty}\left(a_{1} n_{1}^{2}+a_{2} n_{2}^{2}+c^{2}\right)^{-s} .
$$

This series is convergent for $\operatorname{Re} s>2$, and divergent otherwise. Our starting point will be the following summation formula, which is proven in Ref. [22] :

$$
F(\lambda, a)=\sum_{n=-\infty}^{\infty}\left(n^{2}+a^{2}\right)^{-\lambda}=a^{1-2 \lambda}\left[\sqrt{\pi} \frac{\Gamma\left(\lambda-\frac{1}{2}\right)}{\Gamma(\lambda)}+4 \sin \pi \lambda \int_{1}^{\infty} \frac{\left(t^{2}-1\right)^{-\lambda} d t}{\mathrm{e}^{2 \pi a t}-1}\right] .
$$

The series representation of $F$ converges for $\operatorname{Re} \lambda>\frac{1}{2}$, whereas the integral representation is defined for $\operatorname{Re} \lambda<1$.

Let us first use Eq. (A2) to replace the $n_{1}$ summation in Eq. (A1). The result is

$$
A_{2}^{c^{2}}\left(s, a_{1}, a_{2}\right)=\sqrt{\pi} \frac{\Gamma\left(s-\frac{1}{2}\right)}{\Gamma(s)} a_{1}^{-\frac{1}{2}} a_{2}^{\frac{1}{2}-s} F\left(s-\frac{1}{2}, \frac{c}{\sqrt{a_{2}}}\right)
$$




$$
+4 a_{1}^{-s} \sin \pi s \sum_{n=-\infty}^{\infty} a^{1-2 s} \int_{1}^{\infty} \frac{\left(t^{2}-1\right)^{-s} d t}{\mathrm{e}^{2 \pi a t}-1}
$$

where

$$
a=\sqrt{\frac{a_{2} n^{2}+c^{2}}{a_{1}}} .
$$

The function $F\left(s-\frac{1}{2}\right)$ which appears in the first term of Eq. (A3) can in turn be expressed as an integral using Eq. (A2) a second time. The result is

$$
\begin{aligned}
\sqrt{a_{1} a_{2}} A_{2}^{c^{2}}\left(s, a_{1}, a_{2}\right) & =\pi \frac{\Gamma(s-1)}{\Gamma(s)} c^{2(1-s)}+\sqrt{\pi} \frac{\Gamma\left(s-\frac{1}{2}\right)}{\Gamma(s)} \sin \pi\left(s-\frac{1}{2}\right) c^{2(1-s)} \int_{1}^{\infty} \frac{\left(t^{2}-1\right)^{\frac{1}{2}-s} d t}{\mathrm{e}^{2 \pi c t / \sqrt{a_{2}}}-1} \\
& +4 a_{1}^{\frac{1}{2}-s} a_{2}^{\frac{1}{2}} \sin \pi s \sum_{n=-\infty}^{\infty} a^{1-2 s} \int_{1}^{\infty} \frac{\left(t^{2}-1\right)^{-s} d t}{\mathrm{e}^{2 \pi a t}-1}
\end{aligned}
$$

For the purpose of explicitly calculating the mass correction, Eq. (28), we need to evaluate the function $\Gamma(s) A_{2}^{c^{2}}\left(s, a_{1}, a_{2}\right)$ in the neighborhood of $s=0$. The pole term will arise from the factor of $\Gamma(s-1)$ in the first term of Eq. (A5). The remaining two terms will be finite in the $s \rightarrow 0$ limit. The required asymptotic form is

$$
\begin{array}{r}
\sqrt{a_{1} a_{2}} \Gamma(s) A_{2}^{c^{2}}\left(s, a_{1}, a_{2}\right) \sim-\frac{\pi c^{2}}{s}+2 \pi \ln c+8 \pi c^{2} \int_{1}^{\infty} \frac{\left(t^{2}-1\right)^{\frac{1}{2}} d t}{\mathrm{e}^{2 \pi c t / \sqrt{a_{2}}}-1} \\
+4 \sqrt{a_{1} a_{2}} \pi \sum_{n=-\infty}^{\infty} a^{1-2 s} \int_{1}^{\infty} \frac{d t}{\mathrm{e}^{2 \pi a t}-1}, \quad s \rightarrow 0 .
\end{array}
$$

For the purpose of calculating the corrections to the coupling constant, we need to examine the $s \rightarrow 1$ limit of Eq. (A5). There will again be a simple pole coming from the factor of $\Gamma(s-1)$, and all other terms will be regular. However, the $s \rightarrow 1$ limit of the third term on the right hand side of Eq. (A5) requires some care. There is a pole coming from the integral which is cancelled by the $\sin \pi s$ factor. To calculate this explicitly, let

$$
I(s) \equiv \int_{1}^{\infty} \frac{\left(t^{2}-1\right)^{-s} d t}{\mathrm{e}^{2 \pi a t}-1}=\int_{1}^{t_{0}} \frac{\left(t^{2}-1\right)^{-s} d t}{\mathrm{e}^{2 \pi a t}-1}+\int_{t_{0}}^{\infty} \frac{\left(t^{2}-1\right)^{-s} d t}{\mathrm{e}^{2 \pi a t}-1}
$$

where $0<t_{0}-1 \ll 1$. As $s \rightarrow 1$, the contribution of the second integral is finite, so we may write

$$
I(s) \sim \int_{1}^{t_{0}} \frac{(t-1)^{-s}(t+1)^{-s} d t}{\mathrm{e}^{2 \pi a t}-1} \sim \frac{1}{2\left(\mathrm{e}^{2 \pi a}-1\right)} \int_{1}^{t_{0}}(t-1)^{-s} d t .
$$


Furthermore, because $s<1$,

$$
\int_{1}^{t_{0}}(t-1)^{-s} d t=\frac{\left(t_{0}-1\right)^{1-s}}{1-s} \sim \frac{1}{1-s} .
$$

Next, we may use $\sin \pi s \sim \pi(1-s)$ as $s \rightarrow 1$ to write

$$
\sin \pi s I(s) \rightarrow \frac{\pi}{2\left(\mathrm{e}^{2 \pi a}-1\right)}, \quad s \rightarrow 1
$$

Finally, we may combine the above results to yield an expression for $A_{2}^{c^{2}}\left(s, a_{1}, a_{2}\right)$ in the $s \rightarrow 1$ limit:

$$
\begin{aligned}
A_{2}^{c^{2}}\left(s, a_{1}, a_{2}\right) & \sim \frac{\pi}{\sqrt{a_{1} a_{2}}}\left[\frac{1}{s-1}-\ln c^{2}+\cdots\right] \\
& +\frac{4 \pi}{\sqrt{a_{1} a_{2}}} \int_{1}^{\infty} \frac{\left(t^{2}-1\right)^{-\frac{1}{2}} d t}{\mathrm{e}^{2 \pi c t / \sqrt{a_{2}}}-1}+\frac{2 \pi}{a_{1}} \sum_{n=-\infty}^{\infty} \frac{1}{a\left(\mathrm{e}^{2 \pi a}-1\right)},
\end{aligned}
$$

where $a$ is defined by Eq. (A4).

Note that $A_{2}^{c^{2}}\left(s, a_{1}, a_{2}\right)=A_{2}^{c^{2}}\left(s, a_{2}, a_{1}\right)$, whereas the procedure which we have used has obscured this symmetry. Thus, there is an alternative expression for $A_{2}^{c^{2}}\left(s, a_{1}, a_{2}\right)$ in which the roles of $a_{1}$ and of $a_{2}$ are interchanged. We may rewrite Eq. (A11) as

$$
A_{2}^{c^{2}}\left(s, a_{1}, a_{2}\right) \sim \frac{\pi}{\sqrt{a_{1} a_{2}}}\left[\frac{1}{s-1}-\ln c^{2}+\cdots\right]+F_{1}\left(a_{1}, a_{2}\right), \quad s \rightarrow 1,
$$

where $F_{1}\left(a_{1}, a_{2}\right)$ is the finite, $s$-independent part of $A_{2}$ near $s=1$, which can be expressed as

$$
F_{1}\left(a_{1}, a_{2}\right)=\frac{1}{\sqrt{a_{1} a_{2}}}\left[f\left(a_{1}\right)+f\left(a_{2}\right)+R\left(a_{1}, a_{2}\right)\right]
$$

where

$$
f\left(a_{1}\right)=4 \pi \int_{1}^{\infty} \frac{\left(t^{2}-1\right)^{-\frac{1}{2}} d t}{\mathrm{e}^{2 \pi c t / \sqrt{a_{1}}}-1}
$$

and

$$
R\left(a_{1}, a_{2}\right)=2 \pi \sqrt{a_{1}} \sum_{n=-\infty}^{\infty} \frac{1}{\sqrt{a_{1} n^{2}+c^{2}}\left(e^{2 \pi \sqrt{\left(a_{1} n^{2}+c^{2}\right) / a_{2}}}-1\right)}-f\left(a_{2}\right)
$$


or, equivalently,

$$
R\left(a_{1}, a_{2}\right)=2 \pi \sqrt{a_{2}} \sum_{n=-\infty}^{\infty} \frac{1}{\sqrt{a_{2} n^{2}+c^{2}}\left(e^{2 \pi \sqrt{\left(a_{2} n^{2}+c^{2}\right) / a_{1}}}-1\right)}-f\left(a_{1}\right) .
$$

Note that $R\left(a_{1}, a_{2}\right) \rightarrow 0$ as $a_{1} \rightarrow 0$ or as $a_{2} \rightarrow 0$. (The sum in Eq. (A15) may replaced by an integral when $a_{1} \rightarrow 0$, and that in Eq. (A16) may be so replaced when $a_{2} \rightarrow 0$.)

\section{References}

[1] L. Dolan and R. Jackiw, Phys. Rev. D 9, 3320 (1974).

[2] L.H. Ford and T. Yoshimura, Phys.Lett 70A, 89 (1979).

[3] D.J. Toms, Phys.Rev. D 21, 928 (1980); 21, 2805 (1980).

[4] G. Denardo and E. Spalucci, Nucl. Phys. B169, 514 (1980); Y.P. Goncharov, Phys. Lett 91A, 153 (1982); Y. Hosotani, Phys. Lett. 126B, 309 (1983).

[5] G. Jona Lasinio, Nuovo Cim. 34, 1790 (1964); S. Weinberg, Phys. Rev. D 9, 3357 (1974); G. Kennedy, Phys. Rev. D 23, 2884 (1981); G. Denardo and E. Spalucci, Nuovo Cim. 58A, 243 (1981); L.H. Ford, Phys. Rev. D 22, 3003 (1980).

[6] C. J. Isham, Proc. R. Soc. London A362, 383 (1978).

[7] S. Coleman and E. Weinberg, Phys. Rev. D 7, 1888 (1973).

[8] R. Jackiw, Phys. Rev. D 9, 1686 (1974).

[9] M.B. Kislinger and P.D. Morley, Phys. Rev. D 13, 2771 (1976).

[10] H. Matsumoto, I. Ojima and H. Umezawa, Ann. of Phys. (N.Y.) 152, 348 (1984).

[11] D.J. Toms, Ann.of Phys. 129, 334 (1980). 
[12] N.D. Birrell and L.H. Ford, Phys. Rev D 22,330 (1980).

[13] R. Banach, J.Phys.A 13, 1365 (1980).

[14] P. Epstein, Math. Ann. 56, 615 (1902).

[15] Higher Transcendental Functions (Bateman Manuscript Project), edited by A. Erdelyi (McGraw-Hill, New York, 1953) Vol. III, p 195.

[16] A.D. Linde, Rep. Prog. Phys. 42, 390 (1979); D.A. Kirzhnitz and A.D. Linde, Ann. of Phys. 101, 195 (1976).

[17] K. Kirstein, J. Math. Phys. 32, 3008 (1991).

[18] C.G. Bollini, J.J. Giambiagi, and A.G. Domingues, Nuovo Cim. 31, 550 (1964).

[19] E. Speer, J. Math. Phys. 9, 1404 (1968).

[20] A. Higuchi and L. Parker, Phys. Rev. D 37, 2853 (1988).

[21] A.P.C. Malbouisson and N.F. Svaiter, Finite Temperature One-Loop Renormalizability of $\lambda \varphi^{4}$ and the Gross-Neveu Model - The Search for Triviality, preprint.

[22] L.H. Ford, Phys. Rev. D 21, 933 (1980). 